\title{
A small case series of toe sparing surgery: excision of the first metatarsophalangeal joint in diabetic patients to facilitate wound healing
}

\author{
ALOK TIWARI, ${ }^{1}$ IAN WILSON, ${ }^{2}$ MARIANNE DEBRITO, ${ }^{1}$ MIRUNA DAVID, ${ }^{3}$ MUJAHID A SAEED ${ }^{4}$
}

\begin{abstract}
Aim: Various surgical options exist for failed antibiotic treatment of non-healing ulcer or osteomyelitis of the first metatarsophalangeal (MTP) joint. Our aim was to look at the outcome of patients undergoing toe-sparing surgery during excision of the first MTP.

Methods: A prospective series of patients undergoing toesparing surgery of the first MTP joint under one vascular surgeon from 2014 to 2016 and looked after by the multidisciplinary diabetic foot team was studied. All patients undergoing surgery were followed up until wound healing. Complications and time to healing were recorded including any subsequent procedures.

Results: Seven men (mean age $\mathbf{5 6 . 4}$ years) with diabetes underwent this procedure. Mean wound healing time was 9.5 weeks (range 5-16 weeks). All toes were preserved with no new ulcerations and minimal complications.

Conclusion: In selected patients with first MTP joint ulceration and/or osteomyelitis, toe-sparing surgery can be undertaken with excellent results, preserving the toe with fast wound healing and no ischaemia to the adjacent toe.

Br J Diabetes 2018;18:106-109
\end{abstract}

Key words: toe, infection, osteomyelitis, surgery, diabetes mellitus, foot disease

Department of Vascular Surgery, Queen Elizabeth Hospital Birmingham, University Hospitals Birmingham NHS Foundation Trust, Birmingham, UK

2 Department of Podiatry, Queen Elizabeth Hospital Birmingham, University Hospitals Birmingham NHS Foundation Trust, Birmingham, UK 3 Department of Microbiology, Queen Elizabeth Hospital Birmingham, University Hospitals Birmingham NHS Foundation Trust, Birmingham, UK

4 Department of Diabetes, Queen Elizabeth Hospital Birmingham,

University Hospitals Birmingham NHS Foundation Trust, Birmingham, UK

Address for correspondence: Dr Mujahid A Saeed

Queen Elizabeth Hospital Birmingham, University Hospitals Birmingham

NHS Foundation Trust, Mindelsohn Way, Edgbaston, Birmingham

B15 2WB, UK

E-mail: mujahid.saeed@uhb.nhs.uk

http://dx.doi.org/10.15277/bjd.2018.181

\section{Introduction}

In the United Kingdom foot complications due to diabetes cost approximately $\mathrm{f} 650$ million, equivalent to $0.7 \%$ of the National Health Service (NHS) annual budget, ${ }^{1}$ whilst in the USA it is estimated that this is approximately 9-13 billion dollars. ${ }^{2}$ Figures reported in 2015, calculated using new Public Health England data, showed that the annual number of diabetes-related amputations (leg, foot or toe) in England was more than 7,000. ${ }^{3}$ Toe amputations are associated with an increased risk of major amputations due to poor wound healing, further amputation of adjacent toes leading to a cycle of re-intervention and eventually ipsilateral and in some patients contralateral lower extremity amputations. ${ }^{4}$

The currently accepted management of patients with diabetic foot ulceration includes control of infection, ${ }^{5}$ regular debridement and podiatry input, offloading of the ulcer and assessment of the peripheral vasculature and, in addition, control of diabetes and related metabolic abnormalities and advice on smoking cessation. This is usually managed in a multidisciplinary setting. ${ }^{6}$ Failure of this approach may result in toe or leg amputations.

We describe the outcome of seven consecutive patients with diabetes with ulceration affecting the first metatarsophalangeal (MTP) joint who underwent toe-sparing surgery.

\section{Methods}

Patients who attended the multidisciplinary diabetic foot clinic and underwent toe-sparing surgery of the first MTP were identified from a prospective database of all patients undergoing toe surgery under one of the authors (AT). All patients had their vasculature assessed with hand-held Doppler or with a duplex scan if there was any evidence of peripheral vascular disease. Patients who underwent this procedure had either a non-healing ulcer and/or osteomyelitis of the first MTP joint.

All procedures were carried out as day cases under local anaesthetic using a combination of $1 \%$ lidocaine and $0.25 \%$ bupivacaine injected around the base of the toe as a ring block and proximally around the distal metatarsal bone. A medial incision was made over the head of the metatarsal bone and extended proximally and distally incorporating any ulcer margins (Figures $1 \mathrm{a}-\mathrm{c}$ ). The incision was deepened to the bone and the 
Figure 1. Post-operative wound healing following a toe-sparing surgery: (a) day 3; (b) week 4; (c) week 8; and, (d): week 11.
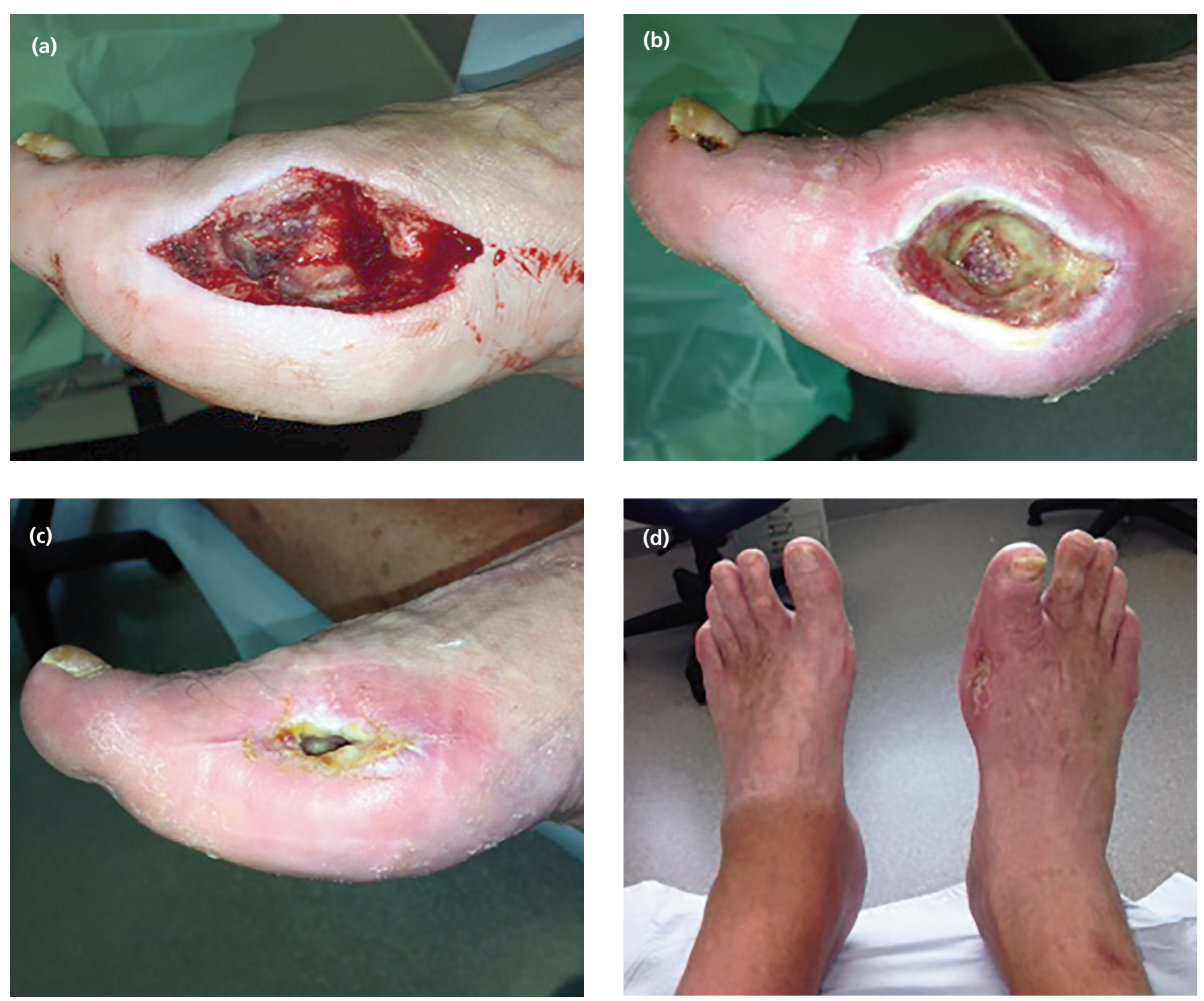

head of the metatarsal and proximal phalanx were excised back to healthy bone. All bone samples were sent for microscopy, culture and sensitivity. If there was extensive soft tissue infection the wound was only partially closed, otherwise interrupted prolene sutures were used to close the skin. The patients were fully ambulant after the procedure wearing only postoperative sandals.

All wounds were reviewed at 48 hours by a podiatrist and a baseline postoperative radiograph was taken (Figure 2). Temporary footwear was provided to prevent flexion of the foot and to offload the operative site. If the bone culture was positive, further antibiotics were continued for 2 weeks or longer (depending on wound healing, soft tissue infection with advice from a microbiologist and also on progression of any infection on radiographs). Patients were followed up by the multidisciplinary diabetic foot team and community podiatry team until complete wound healing (Figure $1 \mathrm{~d}$ ) and resolution of osteomyelitis on serial radiographs. Bespoke footwear was provided via the orthotics department after wound healing.

\section{Results}

Patient demographics, co-morbidities and indications for surgery are summarised in Table 1. All seven patients were male with a mean age of 56.2 years (range 39-79). Two had type 1 diabetes and the other five had type 2 diabetes. Two patients with peripheral vascular disease underwent lower limb revascularisation: one patient had superficial femoral artery angioplasty and the other underwent superficial femoral and popliteal artery angio- 
Figure 2. (a) Pre-, and (b) post-operative plain radiographs of the foot
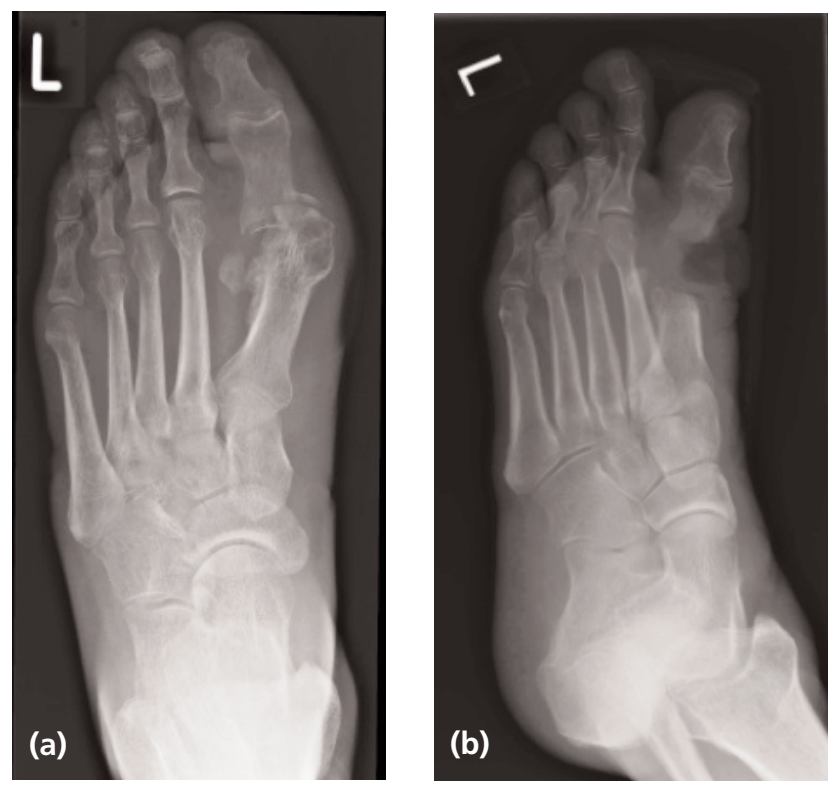

Table 1 Patient demographics, co-morbidities and indications for surgery

\begin{tabular}{lllll}
\hline $\begin{array}{l}\text { Age } \\
\text { (years) }\end{array}$ & $\begin{array}{l}\text { Type of } \\
\text { diabetes } \\
\text { mellitus }\end{array}$ & $\begin{array}{l}\text { Peripheral } \\
\text { vascular } \\
\text { disease }\end{array}$ & $\begin{array}{l}\text { Renal } \\
\text { impairment }\end{array}$ & $\begin{array}{l}\text { Time to } \\
\text { healing } \\
\text { (weeks) }\end{array}$ \\
39 & 2 & No & No & 5 \\
40 & 2 & No & No & 16 \\
57 & 2 & No & Yes & 8 \\
67 & 2 & No & No & 11 \\
60 & 1 & Yes & Yes & 10 \\
53 & 1 & Yes & Yes & 8 \\
79 & 2 & No & No & 10 \\
& & & &
\end{tabular}

plasty before the toe surgery. All patients except one underwent surgery after failed conservative treatment with antimicrobials. One patient had surgery for a non-healing ulcer. The mean time to full wound healing was 9.5 weeks (range 5-16 weeks). One patient's postoperative period was complicated by a methicillinresistant Staphylococcus aureus (MRSA) infection identified on the bone culture. He required intravenous daptomycin to treat this. One patient had subsequent multiple fractures of the heads of the $2 \mathrm{nd} / 3 \mathrm{rd} / 4$ th/5th metatarsals after wound healing due to non-adherence to prescribed footwear. He was treated with a walking cast. There has been no incidence of re-ulceration at the site of the surgical incision. All patients were fully ambulant after wound healing.

To date, none of the patients have had further toe amputations or ulceration at the surgical site (follow up period 9-30 months).

\section{Discussion}

Previous techniques for managing an infected first MTP joint have included ray amputation, excision of bone and insertion of bone cement with external fixation and excision of the metatarsal head through a dorsal incision. The authors do not have experience of using highly purified synthetic calcium sulphate impregnated with antibiotics; however, they are aware of one centre in the UK where this procedure is routinely used. ${ }^{7} \mathrm{~A}$ ray amputation involves excision of the toe and head of the metatarsal. Losing a toe can cause major psychological distress in some patients. In addition, a ray amputation - unless primarily closed - takes a long time to heal. In the series by Nehler et al the mean time to healing was $13 \pm 10$ weeks with complete healing in less than $50 \%$ of the patients, ${ }^{8}$ whilst in a series by Shaikh et al some wounds took up to 30 weeks to heal following a first toe amputation. ${ }^{9}$ In our series, apart from the one patient who developed an infection with MRSA, all patients had ulcer healing by 11 weeks. Paola et al have described a technique which involves excision of the infected bone via a medial incision with excision of the bone, saline irrigation and then placement of antibiotic-loaded bone cement and external fixators. ${ }^{10}$ We have no experience of this technique in our centre.

In this consecutive series of patients we have reviewed outcomes following removal of the first MTP in patients with diabetes in an attempt to offer toe-sparing surgery. The perceived benefits also included supporting patients psychologically with surgery that offered the attraction of preventing loss of the 'big toe'. While we did not formally evaluate the psychological aspect of not losing the toe, verbal feedback was positive. The other advantages of this surgery are that it can be undertaken as a day case thus reducing inpatient stay, the size of the wound negates the need for expensive therapy such as negative pressure wound therapy, community nursing input is reduced and, following healing, footwear requirements are simpler to resolve. The risks of complications to the adjacent toes are reduced, as seen in our series, and long-term biomechanical consequences are minimised. ${ }^{11}$

Our results show that wound healing and preservation of the toes can be achieved. This technique is useful in patients with first MTP joint ulcers or osteomyelitis to preserve the cosmetic appearance of the toes, but also allowing fast wound healing. There is a risk that patients may end up needing to have the toe removed for non-healing wounds, but so far in our series this has not occurred.

The impetus for the toe-sparing surgery described was because two patients who had ulcers overlying the metatarsal head and had failed antibiotic treatment needed ray amputations but were keen to preserve their toes for psychological reasons. The surgical technique described was undertaken with successful results and was offered to other patients needing excision at the MTP joint. The limitation of this study is that patients have been selected from the diabetic foot clinic where the common reason for amputations was failure of multiple antimicrobial therapies. To date, we have had no complications at the surgical site and therefore this technique can be an option in managing non- 


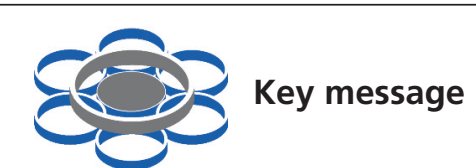

- In selected patients with first metatarsophalangeal joint ulceration and/or osteomyelitis unresponsive to medical therapy, toe sparing surgery can be undertaken with preservation of the hallux and quicker wound healing

healing or infected diabetic foot ulcers at the first MTP joint.

We conclude that, in selected patients with diabetic foot infection/ulcers involving the first MTP joint, toe-sparing surgery can be undertaken to facilitate reduced inpatient stay, shortened wound healing time, early ambulation and with limited risk of ischaemia of the adjacent toe.

\section{Conflict of interest None Funding None}

\section{References}

1. Kerr M. NHS. Footcare for people with diabetes: the economic case for change. https://diabetes-resources-production.s3-eu-west-1.amazonaws. com/diabetes-storage/2017-08/Factsheet\%20Footcare.pdf

2. Rice JB, Desai U, Cummings AK, Birnbaum HG, Skornicki M, Parsons NB. Burden of diabetic foot ulcers for Medicare and private insurers. Diabetes Care 2014:37:651-8. https://doi.org/10.2337/dc13-2176

3. NHS. National Diabetes Foot Care Audit, Third Annual Report, England and
Wales, 14 July 2015 to 31 March 2017, V1.0. 14 March 2018. https://www.hqip.org.uk/wp-content/uploads/2018/03/National-DiabetesFoot-Care-Audit-2014-2017.pdf (accessed 26 April 2018).

4. Murdoch DP, Armstrong DG, Dacus JB, Laughlin TJ, Morgan CB, Lavery LA. The natural history of great toe amputations. J Foot Ankle Surg 1997;36:204-8. https://doi.org/10.1016/S1067-2516(97)80116-0

5. Chisman R, Lowry D, Saeed MA, Tiwari A, David MD. Prescribing antibiotics in diabetic foot infection: what is the role of initial microscopy and culture of tissue samples? Int Wound J 2017;14:685-90. https://doi.org/10.1111/iwj.12674

6. Hingorani A, LaMuraglia GM, Henke $\mathrm{P}$, et al. The management of diabetic foot: a clinical practice guideline by the Society for Vascular Surgery in collaboration with the American Podiatric Medical Association and the Society for Vascular Medicine. J Vasc Surg 2016;63(2 Suppl):3S-21S. https://doi.org/10.1016/j.jvs.2015.10.003

7. Jogia RM, Modha DE, Nisal K, Berrington R, Kong M-F. Use of highly purified synthetic calcium sulfate impregnated with antibiotics for the management of diabetic foot ulcers complicated by osteomyelitis. Diabetes Care 2015;38:e79-80. https://doi.org/10.2337/dc14-3100.

8. Nehler MR, Whitehill TA, Bowers SP, et al. Intermediate-term outcome of primary digit amputations in patients with diabetes mellitus who have forefoot sepsis requiring hospitalization and presumed adequate circulatory status. J Vasc Surg 1999:30:509-17. https://doi.org/10.1016/S0741-5214(99)70078-9

9. Shaikh N, Vaughan P, Varty K, Coll AP, Robinson AH. Outcome of limited forefoot amputation with primary closure in patients with diabetes. Bone Joint J 2013;95-B:1083-7. https://doi.org/10.1302/0301-620X.95B8.31280

10. Dalla Paola L, Carone A, Morisi C, Cardillo S, Pattavina M. Conservative surgical treatment of infected ulceration of the first metatarsophalangeal joint with osteomyelitis in diabetic patients. J Foot Ankle Surg 2015;54:536-40. https://doi.org/10.1053/j.jas.2014.08.004

11. Lavery LA, Lavery DC, Quebedeax-Farnham TL. Increased foot pressures after great toe amputation in diabetes. Diabetes Care 1995;18:1460-2. https://doi.org/10.2337/diacare.18.11.1460 